\title{
QUANTUM CONDUCTANCE FLUCTUATIONS IN 3D BALLISTIC ADIABATIC WIRES
}

\author{
Vladimir I. Fal'ko ${ }^{a, b)}$ and G.B. Lesovik ${ }^{b)}$ \\ a) Max-Planck-Institut für Festkörperforschung, Stuttgart, Germany \\ b) Institute of Solid State Physics, Chernogolovka, 142432 Russia
}

\begin{abstract}
During recent years the transport properties of ballistic adiabatic conductors were extensively studied. The most of the interest in this field has been attracted by the $2 \mathrm{D}$ semiconductor devices, so that the features of a quantum transport in a ballistic adiabatic wire prepared of a bulk metal or semimetal has not been discussed. In the present paper we study the quantum conductance of three dimensional ballistic wires with idealy flat boundaries and show that it obeys fluctuations with the properties quite distinguishable from those of the universal conductance fluctuations (UCF, [1]): both the fluctuations amplitude and the sensitivity of the conductance to the magnetic field flux $\Phi=H S$ penetrated into the sample cross-sectional area $S$ are different and depend on details of the shape of a wire. When the wire has the cross section with the shape of an integrable billiard, conductance fluctuations have the enlarged amplitude $\delta G \sim\left[\left(e^{2} / h\right)^{3} G\right]^{1 / 4}$ and the universal correlation magnetic field $H_{c} \sim \Phi_{0} / S$. When the cross-sectional shape of a wire is non-integrable, the irregular part of a conductance has the $e^{2} / h$ scale whereas the correlation field is reduced to the value of $H_{S} \sim\left(\lambda_{F} / \sqrt{S}\right)^{1 / 2}\left(\Phi_{0} / S\right)$ and the correlation voltage of the nonlinear conductance fluctuations has the scale of $e V_{c} \sim \hbar^{2} / m S \sim E_{F} /\left(S / \lambda_{F}\right)$, where $\lambda_{F}=1 / p_{F}$ is the Fermi wavelength.

The following analysis is based on the application of the Landauer-Buttiker approach. That is, the two-terminal conductance $G$ can be written as $G=$ $\frac{e^{2}}{h} \operatorname{Tr}\left(\hat{t} \hat{t}^{+}\right)$, where $\hat{t}$ is the scattering matrix [2]. In the limit of a ballistic transport in long wires, $t_{n m}=\delta_{n m} \times(0$ or 1$)$ and the separation of variables of the electron motion along and across wire axis reduces the conductance
\end{abstract} formula to the form [3]

$$
G=\frac{e^{2}}{h} N\left(E_{F}\right)
$$


which includes now the number of reflectionless quasi-one-dimensional channels $N\left(E_{F}\right)$. The latter quantity is equal to the number of size-quantized energy levels in a 2D box (with the shape of a wire cross section) below the Fermi level in the system. The main contribution to $N\left(E_{F}\right)$ is determined by the average density of states and depends only on the area $S$ independently of the shape: $N_{0}\left(E_{F}\right) \sim E_{F} /\left(2 \pi \hbar^{2} / 2 m S\right)$. Next, one can distinguish the term which manifests the features of the boundary conditions of the electron wave function along the surcomference $L \sim \sqrt{S}$ of the wire surface. When those are $\psi=0$, a strip $L \times \lambda_{F}$ should be excluded from the cross-sectional area of a sample and, which reduces $N_{0}$ by the number $N_{1}=\theta\left(E_{F} /\left(\hbar^{2} / 2 m L^{2}\right)\right)^{1 / 2}$ [四]. These two terms give us the waveguide analogue to the Sharvin resistance formula [5]. Finally, the exact number of states $N\left(E_{F}\right)$ depends on the Fermi energy irregularly, and its deviation, $\delta N\left(E_{F}\right)$, from the best smoothed approximation gives a subject for a statistical consideration. Altogether, these three contributions can be combined into the conductance

$$
G=\langle G\rangle+\delta G=\frac{e^{2}}{h}\left[\frac{S}{2 \pi \lambda_{F}^{2}}-\frac{L}{\lambda_{F}}\right]+\frac{e^{2}}{h} \delta N\left(E_{F}\right) .
$$

A randomly varying part of a conductance, $\delta G$ will be subject of the following analysis. In Ref. [6] these fluctuations have been called geometrical conductance fluctuations in order to distinguish them from the UCF [1] .

As we know from the quantum billiards theory, the statistics of fluctuations $\delta N\left(E_{F}\right)$ depend on the integrability of the particle motion in a quantum box. According to [7, 8], the fluctuations of the number of states below the Fermi level are the strongest in integrable quantum systems: the systems which possess an additional integral of motion besides the energy. The following reasoning gives a handwaving estimation of this effect. The spectrum $\epsilon(I, n)$ of a particle confined in the integrable box can be imagined as a set of independent level series marked by different values of a quantum number $I$, so that at energies $E \gg h^{2} / m S$ higher than the mean level spacing the total spectrum is locally composed of uncorrelated contributions from different rigid staircases of levels derived from separate one-dimensional Hamiltonians (each corresponding to some value of $I$ ). This means that at the short energy range $\epsilon$ the spectra of integrable systems obey the Poisson type of statistics, $\left\langle(\delta[N(E+\epsilon)-N(E)])^{2}\right\rangle \approx \frac{1}{15}\langle N(E+\epsilon)-N(E)\rangle$ [8], while the width of a spectral interval $\epsilon$ is small enough to consider all levels $\epsilon(I, n)$ inside as 
taken independently from different series $I$. Otherwise, the mean square $\left\langle(\delta[N(E+\epsilon)-N(E)])^{2}\right\rangle$ is restricted by the number $I_{\max }$ of independent level series contributing to the spectrum formation, since each of them has a strong internal rigidity. In the case of a free particle moving in a $2 \mathrm{D}$ box, $\epsilon(I, n) \propto\left(I^{2}, n^{2}\right)$, so that the above-mentioned Poisson law is applicable only if $\epsilon<\epsilon_{\max } \sim E_{F} / \sqrt{S / \lambda_{F}^{2}}$. Beyond this scale, the amplitude of spectral fluctuations is saturated, what can be estimated as $\left\langle\left(\delta\left[N\left(E_{F}+\epsilon\right)-N\left(E_{F}\right)\right]\right)^{2}\right\rangle$ $=\left\langle\delta N\left(E_{F}\right)^{2}\right\rangle \sim N\left(E_{F}\right)^{1 / 2}$. It is amusing to note that the problem of calculation of $\delta N\left(E_{F}\right)$ in rectangular billiards is familiar to the number-theory as a problem of an accuracy of the best smoothed series approximation of a number of square lattice vortices inside an ellipse. One of the best numbertheory results 9] has predicted $\left\langle\delta N^{2}\right\rangle \propto\langle N(E)\rangle^{\theta}$, where $0.6416 \geq \theta \geq 1 / 2$, which agrees with the above qualitative reasoning.

All this gives an estimation of the mean square value of the conductance fluctuations $\delta G$ in the wire with an integrable cross sectional shape,

$$
\left\langle\delta G^{2}\right\rangle=\beta \alpha\left[\left(\frac{e^{2}}{h}\right)^{3} G\right]^{\frac{1}{2}} .
$$

The scale of these fluctuations can exceed the quantum $\frac{e^{2}}{h}$. Here, $\alpha$ is a specific geometrical factor (for instance, $\alpha=0.095$ in a rectangle with practically equal sides) and $\beta$ accounts for the symmetry induced degeneracy of states.

The spectra of non-integrable systems are much more rigid, and the fluctuations in them are rather weak. In terms of the interaction of levels, this results from the repulsion between all of them (not only within each spectral series, as in the case of integrable billiards). The limiting case of shuc levels statistics is given by that of chaotic billiards [8], i.e., the quantity $\delta N\left(E_{F}\right)$ obeys some kind of a saturated Wigner-Dyson law [10. Therefore, the amplitude of geometrical conductance fluctuations in wires of an arbitrary shape can be estimated as

$$
\left\langle\delta G^{2}\right\rangle=\beta\left(\frac{e^{2}}{\pi h}\right)^{2} \ln \left(\ln \frac{G h}{e^{2}}\right) .
$$

Fluctuations of nonlinear conductance. Since neither the shape nor the Fermi level can be easily varied in 3D metallic wires, the most natural 
possibility to observe the spectral fluctuations of the transverse motion in an adiabatic wire consists in the studies of its diferential (nolinear) conductance $d I(V) / d V$. In the adiabatic regime, this quantity can be expressed in terms of a number of transmitted waveguide modes [11] as

$$
\frac{d I(V)}{d V}=\frac{e^{2}}{2 h}\left[N\left(E_{F}+\frac{e V}{2}\right)+N\left(E_{F}-\frac{e V}{2}\right)\right] .
$$

One can see from this equation that the voltage dependence of $d I(V) / d V$ just follows the local (in energy) fluctuations of the number of size-quantized states in the interval $\mathrm{eV}$ near the Fermi level. In a constriction with the cross section presenting completely integrable billiard, this value undergoes fluctuations with the Poisson statistics at low voltages $V<V_{c}=\epsilon_{\max } / e$. When $V \geq V_{c}$, the amplitude of fluctuations saturates at the value described by Eq. (2) and then $V_{c}$ plays the role of the correlation voltage of these fluctuations. Therefore, in the adaibatic contact with an integrable crosssectional shape

$$
\left\langle\left[\frac{d I(V)}{d V}-G\right]^{2}\right\rangle=\left\{\begin{array}{c}
\frac{1}{15}\left(\frac{e V}{E_{F}}\right) \frac{e^{2}}{h} G, e V<\epsilon_{\max } \\
\gamma \sqrt{\left(\frac{e^{2}}{h}\right)^{3} G}, e V>\epsilon_{\max }
\end{array} .\right.
$$

In non-integrable (i.e., chaotic) systems the spectral fluctuations are weaker [8, 10],

$$
\left\langle\left[\frac{d I(V)}{d V}-G\right]^{2}\right\rangle=3 \beta\left(\frac{e^{2}}{\pi h}\right)^{2}\left\{\begin{array}{c}
\ln \left(\frac{V G h}{e E_{F}}\right), e V<\epsilon_{\max }^{\prime} \\
\ln \left(\ln \left(\frac{G h}{e^{2}}\right)\right), e V>\epsilon_{\max }^{\prime}
\end{array} .\right.
$$

The correlation voltage which one could assign to these fluctuations is determined by the mean level spacing $\hbar^{2} / m S$ and has the form

$$
V_{c}=\frac{1}{e} \frac{E_{F}}{S / \lambda_{F}^{2}}
$$

It is interesting to note that this result differs from the time-of-flight estimation which one could get after replacing the mean free path by the sample length in the formulae valid for diffusive conductors [12]. 
Magnetoconductance fluctuations. Another possibility to observe the geometrical conductance fluctuation consists in an application of a magnetric field oriented along the axis of a wire. In what follows, we distinguish three cases of different cross-sectional shapes of a wire.

Let us first consider the system which is integrable and retains this property also in an applied magnetic field (the disk obviously belongs to this class). The external magnetic field shifts series of levels marked by different value of the angular momentum $I$, one with respect to another. When this shift, $\frac{\hbar e}{m c} H I \sim H \frac{\hbar e}{m c} \sqrt{E_{F} /\left(\hbar^{2} / 2 m S\right)}$, becomes comparable to the mean intraseries level spacing, $\sqrt{E_{F} \hbar^{2} / 2 m S}$, the statistical configuration of the Poisson distribution of levels is renewed, which produces a random magnetoconductance variation of the order of what is represented in Eq. (2) $(\beta=1)$. The characteristic scale of a sufficient magnetic field is determined by the flux quantum penetrated through the sample cross-sectional area, $H_{c} S \sim h c / e$, which means that the important physical quantity - the correlation magnetic field of fluctuations [1] - is similar to that in UCF. The visual difference between UCF and geometrical fluctuations is only in their amplitudes. The geometrical fluctuations are enlarged parametrically in thick cylindrical wires, what has been observed in [13] in numerical simulations. On the other hand, these fluctuations can be viewed (even inside the correlation field $H_{c}$ ) as a series of randomly distributed $e^{2} / h$ conductance steps with the characteristic spacing of $\Phi_{S} \sim \frac{\Phi_{0} / \sqrt{\alpha}}{\sqrt[4]{N\left(E_{F}\right)}} \sim \Phi_{0}\left[\frac{2 e^{2} / h}{\alpha^{2} G}\right]^{\frac{1}{4}}$.

Of course, one should realize that the wires with the cross-sectional shape which is integrable at any magnetic field gives us an exclusive example. Magnetoconductance fluctuations in wires with a non-integrable cross-sectional shape (see Eq. (3)) have a much lower amplitude what makes them more similar to the UCF. Nevertheless, they still possess one feature specific to the ballistic adiabatic system. That is, the non-integrable quantum box spectrum is much more sensitive to the magnetic field variation that it takes place in open diffusive conductors, and the penetration of a flux

$$
\Phi_{S}=\sqrt{\frac{\lambda_{F}}{\sqrt{S}}} \frac{h c}{e} \ll \Phi_{0}
$$

is already enough for renewing the realization of the levels configuration [14]. In a chaotic billard, all the classical electron trajectories are infinitely long 
and cover the whole fixed-energy thorus in the phase space. In semiclassics, the length of such trajectories is limited, since two point of the phase space which reach into the same unit volume $d p_{i} d x_{i} \sim h$ are indistinguishable. Therefore, the length of a semiclassical trajectory in a box of characteristic dimensions $L \sim \sqrt{S}$ should be cut when the inverse time of flight along it, $h \sqrt{S} / v_{F}$ will be comparable with the mean level spacing $\Delta \sim \hbar^{2} / m S$ and characteristic traces in the real space contain $\sim \sqrt{S} / \lambda_{F}$ closed loops each encircling an area $\sim S$ of a random sign. The oriented encircled area of a chaotic trajectory can be estimated as $S^{3 / 2} / \lambda_{F}$ which produces the characteristic correlation magnetic field $H_{c} \sim \Phi_{S} / S$. It would be interesting to note in this connection that the features of the conductance fluctuations found in ballistic silver micro-contacts [15] are quite similar to those of wires with a non-itegrable shape described by Eqs. (6,7), though the observed amplitudes were much smaller than $e^{2} / h$ scale.

Finally, there exists a class of systems which are integrable (or partly integrable [8]) at $H=0$ and loose this property after an application of a magnetic filed. Fot instance, we can mention the structures with a rectangular cross-sectional shape where the first penetrated flux quantum drastically changes the level statistics: from the Poisson type at $\Phi=H S<\Phi_{0}$ to the Wigner-Dyson type at higher fields. This should induce a low-field magnetoconductance of a random sign with the amplitude estimated by Eq. (2), whereas the following increase of a produces fluctuations with the amplitude $\frac{e^{2}}{h}$ and at the scale of $\Phi_{S}$ (instead of $\Phi_{0}$ observed in the UCF). The monocrystalline microwires with perfect facets (whiskers) grown of the semimetallic material would be the best candidates to show this kind of behavior.

We are greateful to P. Wyder and A. Jansen for helpful discussions.

\section{References}

[1] B.L. Altshuler, JETP Lett. 41, 648 (1985); B.L. Altshuler and D.E. Khmel'nitskii, ibid. 42, 359 (1985); P.A. Lee and A.D. Stone, Phys. Rev. Lett. 55, 1622 (1985); A.D. Stone, ibid. 54, 2692 (1985)

[2] M. Buttiker et al, Phys. Rev. B 31, 6207 (1985)

[3] B.J. van Wees et al, Phys Rev Lett 60, 848 (1988) 
[4] H. Weyl, "Gruppentheorie in Quantenmechanik", Hirzel, Leipzig 1931

[5] Yu. V. Sharvin, Sov Phys JETP 21, 655, (1965)

[6] V.I. Fal'ko and G.B. Lesovik, Solid State Commun. 84, 835 (1992)

[7] O. Bohigas et al, Phys. Rev. Lett. 52, 1 (1984); G. Casati et al, ibid. $\mathbf{5 4}, 1350(1985)$

[8] M. Berry, Proc. Roy. Soc. Lond. A 400, 229 (1985); M. Gutzwiller, "Chaos in Classical and Quantum Mechanics", Springer-Verlag, Berlin 1990

[9] V. Vinogradov, Bull. Ac. Sci. USSR Ser. VIII 3, 313 (1932); H.P. Baltes and E.R. Hilf, "Spectra of Finite Systems", B.I.-Wissenshaftsverlag, Mannheim 1976

[10] E.P. Wigner, SIAM Review 9, (1967), 1; M.L. Mehta, "Random Matrices", Academic Press, NY 1967

[11] L.I. Glazman and A.V. Khaetskii, JETP Lett 48, 591 (1988)

[12] D.E Khmelnitskii and A.I. Larkin, Phys. Scr. T 14, 4 (1986);

[13] E.N. Bogachek et al, Phys. Rev. B 47, 16635 (1993)

[14] K.B. Efetov, Adv. Phys. 32, 53 (1983); R. Jalabert et al, Phys. Rev. Lett. 65, 2442 (1990); A. Szafer and B.L. Altshuler, ibid. 70, 587 (1993)

[15] P.A. Holweg et al, Phys. Rev. Lett. 67, 2549 (1991); P.A. Holweg et al, Phys. Rev. B 48, 2479 (1993) 